\title{
Trombosis venosa superficial extensa y síndrome de Behçet
}

\author{
Neera Toledo-Samaniego, Francisco Galeano-Valle, Eva Cervilla-Muñoz, Sergio Moragón-Ledesma, Pablo Demelo-Rodríguez \\ Servicio de Medicina Interna, Hospital General Universitario Gregorio Marañón, Madrid, España
}

\section{Recibido: 25/10/2020 \\ Aceptado: 08/12/2020 \\ En línea: 30/04/2021}

Citar como: Toledo-Samaniego N., Galeano-Valle F., Cervilla-Muñoz E, Moragón-Ledesma S., Demelo-Rodríguez P. Trombosis venosa superficial extensa y síndrome de Behçet. Rev Esp Casos Clin Med Intern (RECCMI). 2021 (abril); 6(1): 7-9. doi: 10.32818/reccmi.a6n1a3.

Cite this as: Toledo-Samaniego N., Galeano-Valle F., Cervilla-Muñoz E, Moragón-Ledesma S., Demelo-Rodríguez P. Extensive superficial vein thrombosis and Behçet's syndrome. Rev Esp Casos Clin Med Intern (RECCMI). 2021 (April); 6(1): 7-9. doi: 10.32818/reccmi.a6n1a3.

\begin{tabular}{l} 
Palabras clave \\
\hline$\triangleright$ Enfermedad \\
$\quad$ tromboembólica venosa \\
$\triangleright \quad$ Trombosis venosa \\
$\quad$ superficial \\
$\triangleright$ Síndrome de Behçet \\
$\triangleright$ Anticoagulación
\end{tabular}

\section{Keywords}

$\triangleright$ Venous thrombosis disease

$\triangleright$ Superficial venous thrombosis

$\triangleright$ Behçet Syndrome

$\triangleright$ Anticoagulation

\begin{abstract}
Resumen
La trombosis venosa superficial es frecuente; su principal factor de riesgo es la presencia de venas varicosas en miembros inferiores, aunque también puede ocurrir en otros estados protrombóticos. El síndrome de Behçet es una enfermedad crónica, caracterizada por aftas orales y genitales y afectación ocular, que también puede aparecer con manifestaciones vasculares, actual criterio diagnóstico.

Mujer de 62 años, con antecedentes de episodios de aftas orales y uveítis, acude a Urgencias por trombosis venosa superficial; cumple los criterios para el diagnóstico de síndrome de Behçet. El tratamiento de las trombosis venosas en el síndrome de Behçet es la inmunosupresión. Se inició tratamiento con azatioprina sin recurrencias posteriores.

Abstract
Superficial venous thrombosis is frequent; its main risk factor is variceal veins' presence in lower limbs, although
it can occur in other prothrombotic states. Behçet syndrome is a chronic disease characterized by recurrent oral
and genital aphthous and ocular involvement; vascular involvement may also appear, which is a diagnostic
criterion.
A 62-year-old woman, with recurrent oral and ocular involvement (uveitis) was admitted in the emergency
room because of superficial venous thrombosis, meeting diagnostic criteria for Behçet syndrome. The treatment
of vein thrombosis in Behçet syndrome is immunosuppression. We started azathioprine, and no recurrences
were registered.
\end{abstract}

\section{Puntos destacados}

$\triangleright$ El síndrome de Behçet es una enfermedad infrecuente que puede cursar con manifestaciones vasculares, principalmente trombosis venosas, siendo la trombosis venosa superficial la más frecuente.

$\triangleright$ La aparición de trombosis venosa en un contexto clínico adecuado debe hacer sospechar la presencia de un SB.

\section{Introducción}

La trombosis venosa superficial (TVS) es una entidad frecuente, con una prevalencia estimada en 1 por 1000 habitantes $^{1}$. El principal factor de riesgo para su desarrollo es la presencia de venas varicosas en miembros inferiores. Su tratamiento se dirige al alivio sintomático $y$, en algunos casos, se prescribe un tratamiento anticoagulante.

El síndrome de Behçet (SB) es una enfermedad multisistémica recurrente que presenta una distribución geográfica típica en la cuenca del Mediterráneo, con una baja prevalencia en nuestro medio ${ }^{2}\left(<50 / 10^{5}\right)$. Hasta en un $40 \%$ de los casos pueden aparecer manifestaciones vasculares: las más frecuentes son las trombosis venosas ${ }^{2}$, que se caracterizan por presentar localizaciones extensas, recurrencias frecuentes y que no responden al tratamiento anticoagulante, siendo de primera elección el tratamiento inmunosupresor ${ }^{3}$.

Presentamos el caso de una mujer de 62 años con trombosis venosa superficial extensa la cual fue determinante para establecer el diagnóstico clínico de SB. Se han seguido para la publicación el protocolo del Comité de Ética e Investigación Clínica del centro respetando la privacidad del paciente.

\section{Caso clínico}

Antecedentes personales

Mujer de 62 años con antecedentes personales de hipertensión arterial, dislipemia, hipotiroidismo y presencia de venas varicosas en miembro inferior de- 
recho. La paciente no tiene historia de tabaquismo y el índice de masa corporal es de $24 \mathrm{~kg} / \mathrm{m}^{2}$. Tomaba lisinopril, atorvastatina y levotiroxina. Presentaba episodios recurrentes de aftas orales dolorosas, episodios de uveítis desde hacía 4 años y positividad para el alelo HLA-B51. Estaba en seguimiento en consultas de Reumatología.

\section{Historia clínica y exploración física}

Acude a Urgencias por dos semanas de evolución de dolor y pesadez en la zona posterior de la pierna derecha, con induración y eritema en la parte posterior de dicha extremidad, desde la región gemelar hasta región proximal del muslo. No había presentado en las semanas previas inmovilización prolongada, traumatismos o cirugías.

Paciente en buenas condiciones generales, afebril, apreciándose venas varicosas sin signos de complicación y un cordón varicoso indurado y doloroso a la palpación en la cara posterior del gemelo derecho extendiéndose proximalmente hacia la cara postero-interna del muslo.

El resto de la exploración física era normal.

\section{Pruebas complementarias}

En el análisis de sangre destaca hemograma (hemoglobina 13,0 g/dL; leucocitos 6500/ $\mu \mathrm{L}$; plaquetas $224000 / \mu \mathrm{L}$ ), iones en rango normal, enzimas de citolisis y colestasis sin alteraciones (alanino aminotransferasa $16 \mathrm{U} / \mathrm{L}$; bilirrubina 0,5 mg/dL; gamma-glutamil transferasa $117 \mathrm{U} / \mathrm{L}$; fosfatasa alcalina $43 \mathrm{U} / \mathrm{L}$ ), función renal preservada, fibrinógeno $439 \mathrm{mg} / \mathrm{dL}$, proteína $C$ reactiva $<0,4 \mathrm{mg} / \mathrm{dL}$ y dímero D de 300 ng/mL (límite superior $250 \mathrm{ng} / \mathrm{mL}$ ).

Una ecografía-doppler de la extremidad afecta mostró en el sistema venoso superficial una vena colateral con contenido ecogénico intraluminal, ausencia de colapso a las maniobras de compresión y ausencia de flujo Doppler, desde el tercio medio de la región posterior del músculo gastrocnemia derecho extendiéndose cranealmente hasta región proximal del muslo (> 20 cm de extensión).

\section{Evolución}

Las aftas orales podrían explicarse en el contexto de una infección vírica (virus herpes simple, virus de la inmunodeficiencia humana, etc.) o por enfermedades inflamatorias sistémicas, como la enfermedad de Crohn o el SB. Tanto esta como una artritis reactiva o la sarcoidosis pueden tener una uveítis como manifestación ocular. La TVS puede ocurrir en el seno de infecciones, enfermedades inflamatorias, patología oncológica o asociada a catéteres.

En este caso, la aparición de la TVS unido a los antecedentes de episodios de uveítis y de aftas orales dolorosas recurrentes permitió establecer el diagnóstico de SB. Se inició tratamiento con fondaparinux 2,5 mg subcutáneo 1 inyección al día durante 45 días, prednisona a dosis de 0,5 mg/kg/día y azatioprina 100 mg/día. La paciente presentó una evolución clínica favorable sin nuevas recurrencias de trombosis tras dos años de seguimiento.

\section{Diagnóstico}

Trombosis venosa superficial extensa secundaria a síndrome de Behçet.

\section{Discusión y conclusión}

La TVS es una entidad relativamente común en nuestro medio, que comparte factores de riesgo con la trombosis venosa profunda (edad, inmovilización, obesidad, cáncer activo, estados protrombóticos y embarazo, entre otros), aunque su principal factor de riesgo es la presencia de venas varicosas en extremidades inferiores, encontrándose hasta en un $80-90 \%$ de los pacientes con TVS 4 .

El tratamiento de la TVS es sintomático, dirigido al alivio del dolor y a la prevención de complicaciones para evitar la extensión hacia el sistema venoso profundo. Se recomienda terapia anticoagulante si la longitud del trombo es mayor a 4-5 cm o está próximo al cayado de la safena. En el ensayo clínico CALISTO 5 se demostró la eficacia de fondaparinux 2,5 mg subcutánea al día durante 6 semanas frente al placebo; se disminuía la aparición de eventos trombóticos sintomáticos (trombosis venosa profunda [TVP] y embolia pulmonar) y mortalidad por todas las causas.

El SB es una enfermedad multisistémica con una distribución geográfica alrededor de la ruta de la seda, que se caracteriza por lesiones en las mucosas (tanto oral como genital), además de posible afectación ocular, vascular, neurológica o gastrointestinal ${ }^{6}$. Las manifestaciones vasculares pueden ocurrir hasta en un $40 \%$ de los pacientes con SB ${ }^{7}$, siendo las trombosis venosas más frecuentes que las arteriales $y$, dentro de ellas, las TVP y las TVS (61,5\% y 19,2\% respectivamente $\left.{ }^{6}\right)$. La patogenia del trombo en el SB es predominantemente inflamatoria ${ }^{8}$ lo cual explica la escasa eficacia de la anticoagulación en el paciente con SB y trombosis y, por lo tanto, el tratamiento de elección según la European League Against Rheumatism (EULAR) es la inmunosupresión ${ }^{3}$.

El diagnóstico de trombosis venosa debe hacer sospechar en la posibilidad de un SB cuando se presenta en pacientes jóvenes, trombosis extensas, escasa respuesta al tratamiento anticoagulante o cuando presenta otros síntomas sugestivos de SB. El diagnóstico del SB es clínico: se basa en los criterios establecidos en 1990 (International Study Group for Behçet's Disease) y actualizados posteriormente en 2013 (International Criteria for Behçet's Disease). Se incluye las manifestaciones vasculares como un criterio diagnóstico en estos últimos.

Un estudio observacional en una cohorte de pacientes con manifestaciones vasculares en SB publicado recientemente por nuestro grupo ${ }^{6}$ mostraba que hasta un $80 \%$ de los pacientes con SB pueden no estar diagnosticados cuando se presenta la manifestación vascular. Este tipo de manifestaciones son las que llevan al diagnóstico del SB en un 40\%, quedando un porcentaje alto sin diagnosticar y, por tanto, sin tratamiento, con alta probabilidad de recurrencia de trombosis con la morbimortalidad que asocia.

Como conclusión, queremos destacar la importancia de una correcta anamnesis y sospecha clínica de SB ante un evento vascular en un paciente con clínica sugestiva de SB, pudiendo ser la primera manifestación del SB y/o la que conlleve al correcto diagnóstico y su posterior tratamiento.

\section{Bibliografía}

1. Nasr H, Scriven JM. Superficial thrombophlebitis (superficial venous thrombosis). BMJ. 2015; 350: h2039. doi: 10.1136/bmj.h2039.

2. Seyahi E. Phenotypes in Behçet's syndrome. Intern Emerg Med. 2019; 14(5): 677-689. doi: 10.1007/s11739-019-02046-y.

3. Hatemi G, Christensen R, Bang D, Bodaghi B, Celik AF, Fortune F, et al. 2018 update of the EULAR recommendations for the management of Behçet's syndrome. Ann Rheum Dis. 2018; 77(6): 808-818. doi: 10.1136/annrheumdis-2018-213225.

4. Cosmi B. Management of superficial vein thrombosis. J Thromb Haemost. 2015; 13(7): 1175-83. doi: 10.1111/jth.12986.

5. Decousus H, Prandoni P, Mismetti P, Bauersachs RM, Boda Z, Brenner B, et al: CALISTO Study Group. Fondaparinux for the treatment of superficial-vein 
thrombosis in the legs. N Engl J Med. 2010; 363(13): 1222-32. doi: 10.1056/ NEJMoa0912072.

6. Toledo-Samaniego N, Galeano-Valle F, Pinilla-Llorente B, Del-Toro-Cervera J, Marra A, Proietti M, et al. Clinical features and management of venous thromboembolism in patients with Behçet's syndrome: a single-center case-control study. Intern Emerg Med. 2020; 15(4): 635-644. doi: 10.1007/ s11739-019-02237-7.
7. Emmi G, Bettiol A, Silvestri E, Di Scala G, Becatti M, Fiorillo C, et al. Vascular Behçet's syndrome: an update. Intern Emerg Med. 2019; 14(5): 645-652. doi: 10.1007/s11739-018-1991-y.

8. Emmi G, Becatti M, Bettiol A, Hatemi G, Prisco D, Fiorillo C. Behçet's Syndrome as a Model of Thrombo-Inflammation: The Role of Neutrophils. Front Immunol. 2019; 10: 1085. doi: 10.3389/fimmu.2019.01085. 